\title{
Terahertz bandwidth photonic Hilbert transformers and implementations in ultra wideband single-sideband filters
}

\author{
Chaotan Sima ${ }^{(1)}$, James C. Gates ${ }^{(1)}$, Christopher Holmes ${ }^{(1)}$, Michalis N. Zervas ${ }^{(1)}$, Peter G. R. Smith ${ }^{(1)}$ \\ (1) Optoelectronics Research Centre, University of Southampton, Southampton, SO17 1BJ, UK, \\ Chaotan.Sima@soton.ac.uk
}

\begin{abstract}
Planar Bragg grating based photonic Hilbert transformers (PHTs) with THz bandwidths are proposed and practically demonstrated. An X-coupler, PHT, and a flat-top reflector are incorporated, demonstrating $2 \mathrm{THz}$ all-optical single-sideband filters. Devices are fabricated via a direct UV grating writing technique on a silica-on-silicon platform.
\end{abstract}

\section{Introduction}

In the electronic domain Hilbert transform technology has been applied to various application areas such as single-sideband (SSB) modulation for improving performance and enhancing bandwidth efficiency ${ }^{1}$. In the optical domain photonic Hilbert transformers (PHTs) show the potential to provide large bandwidths at high speeds for applications such as broadband microwave signal characterisation ${ }^{2}$ and all-optical SSB filtering ${ }^{3,4}$.

PHTs have been practically implemented via various methods, e.g. using multi-tap sampled fibre Bragg gratings (FBGs) ${ }^{5}$, phase-shifted and apodized $\mathrm{FBGs}^{6,7}$, and ring resonators ${ }^{8}$. These current solutions are limited to operation bandwidths less than $100 \mathrm{GHz}$ due to the intrinsic complexity of approaches via discrete components or the restrictions of current FBG fabrication ${ }^{4}$. Terahertz bandwidth PHTs have been numerically modelled using long period gratings and core/cladding modes interaction ${ }^{10}$, nevertheless, the complexity of this approach provides a significant challenge to practically realizing such a device.

In this work, we physically demonstrate a direct and simple route to realize Bragg grating based PHTs with operation bandwidths up to 2 $\mathrm{THz}(\sim 16 \mathrm{~nm})$, over an order of magnitude larger than any previously reported experimental approaches. The PHTs have been integrated with couplers and reflectors to implement ultra wideband all-optical SSB filters. We use a silicaon-silicon planar format $^{10}$, which provides a route to realize compact optical modules for integrated optical applications.

\section{Operation principle}

The conventional Hilbert transform function $\mathrm{H}(\omega)$ in frequency domain is defined as ${ }^{1}$ :

$$
H(\omega)=-j \operatorname{sgn}(\omega)
$$

where $\omega$ is the angular frequency and $\operatorname{sgn}(\omega)$ is the sign function (which is +1 for $\omega>0,0$ for $\omega=0$, and -1 otherwise).

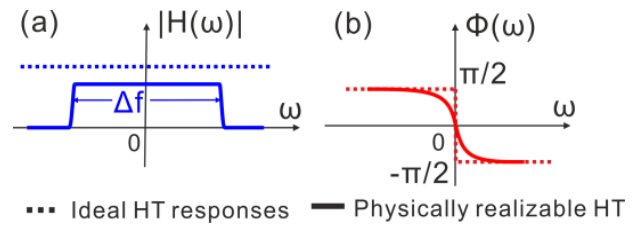

Fig. 1: (a) Amplitude and (b) phase responses of a physically realisable $\mathrm{HT}$ (solid lines) compared to those of the ideal HT (dashed lines).

In any real implementation of a Hilbert transform, the output signal is a delayed and somewhat distorted HT of the input signal. As a consequence the frequency response of the HT will be band-limited with a bandwidth $\Delta f$ with a smooth alteration in phase, as illustrated in Fig. 1. To physically realize these devices, the practical grating apodization profile is ${ }^{6}$ :

$$
\Delta n(z) \propto \frac{\sin ^{2}\left[\pi n_{e f f} \Delta f\left(z-z_{0}\right) / c\right]}{z-z_{0}}
$$

where $n_{\text {eff }}$ is the grating effective refractive index, $\mathrm{c}$ is the light speed in vacuum, $\mathrm{z}$ is the grating length, $z_{0}$ is the zero-crossing point in the apodization function, and $\Delta \mathrm{f}$ is the operative bandwidth. From Eq. (2), the full operation spectral bandwidth of the PHT is inversely proportion to the period of the apodization profile $\Delta \mathrm{z}$, (also refers to the zero-to-zero width of the sidelobe in the apodization profile ${ }^{7}$ ) as shown in Fig. 2(a), given as:

$$
\Delta f=\frac{c}{n_{e f f} \Delta z}
$$

Therefore a broader processing bandwidth can be obtained using narrower apodization sidelobe. A transfer matrix method was employed for modeling the device output with the proposed structure, assuming the grating length is $10 \mathrm{~mm}, \mathrm{n}_{\text {eff }}=1.45$ and the refractive index modulation depth was $\Delta n=9 \times 10^{-4}$.

Figure 2(a) presents the central section of the apodization profile for $1 \mathrm{THz}$ bandwidth PHT scenario, showing the sidelobe width $\Delta z$ and the $\pi$ phase shift. Figure 2(b) illustrates the 
reflectivity spectra of the proposed Bragg gratings, with bandwidths of $1 \mathrm{THz}, 1.5 \mathrm{THz}$ and $2 \mathrm{THz}$ respectively; thus, the apodization profile period $\Delta z$ were around $207 \mu \mathrm{m}, 138 \mu \mathrm{m}$ and 104 $\mu \mathrm{m}$, according to Eq. (3).

\section{Experimental demonstration}
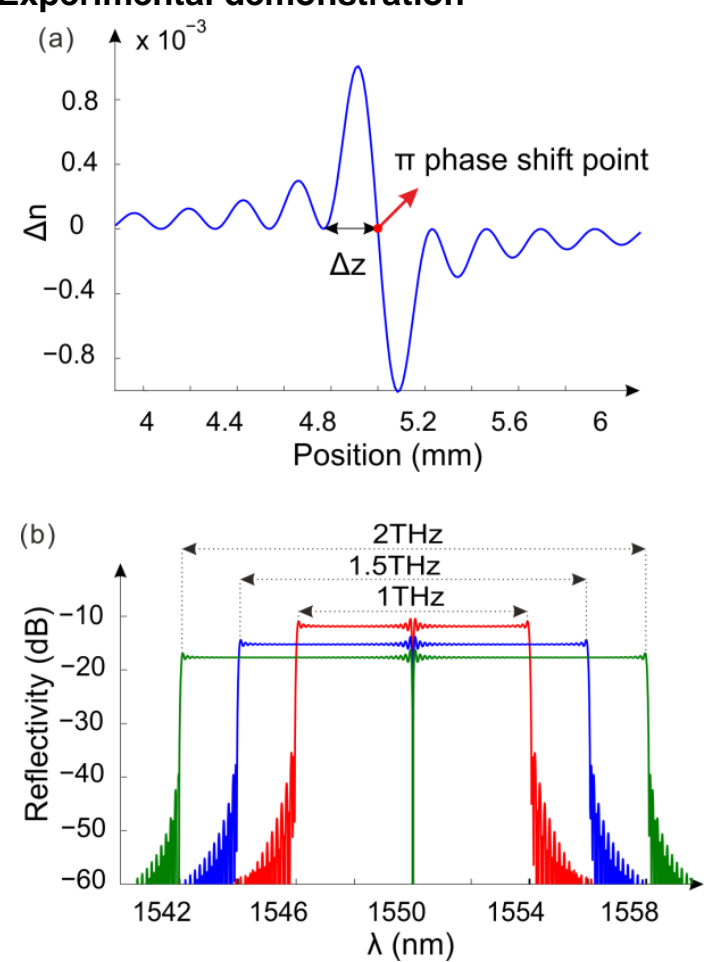

Fig. 2:(a) The apodization profile in the central section of the Bragg grating for $1 \mathrm{THz}$ bandwidth PHT; (b) Modelling of the amplitude responses of a series of PHTs with terahertz bandwidths.

For current FBG fabrication technologies using phase masks, the UV exposure spot allows writing grating apodization profiles with spatial resolutions about $100 \mu \mathrm{m}$ and hence previously fabricated Bragg grating based PHTs suffered the operation bandwidth limitations $<100 \mathrm{GHz}$. To overcome the restrictions and to obtain larger bandwidths, we use the alternative fabrication approach.

The proposed planar structures were fabricated using the direct UV grating writing technique $^{10}$, shown in Fig. 3. This method involves focusing two crossed laser beams $(\lambda=$ $244 \mathrm{~nm}$ ) into the photosensitive core of a planar sample. Precise translation of the sample and modulation of the interference pattern creates grating structures and simultaneously defines the channel waveguide. This spot size is $\sim 6 \mu \mathrm{m}$ in diameter, providing the unique ability over traditional FBG to manipulate the grating's structure at the micron level.

The fabricated devices had a three-layer silica-on-silicon substrate, containing a thermal oxide layer (lower clad), core and an upper clad.
The Bragg gratings sections were all $10 \mathrm{~mm}$ long, the effective refractive index of the mode within the gratings was 1.447 , and the maximum refractive index modulation depth was $\Delta n=$ $0.9 \times 10^{-3}$. Figure 4 shows the measured (a) reflectivity and (b) relative group delay data of the fabricated devices with terahertz bandwidths. Grating reflectivity was measured using an optical spectrum analyzer (OSA), 3dB coupler and an erbium doped fiber amplified spontaneous emission (ASE) source. The measurement system has a noise floor of about $-30 \mathrm{~dB}$. Due to lack of availability of a direct optical phase measurement system, a modulation phase-shift method ${ }^{11}$ was used to obtain relative group delay data instead. In Fig. 4(a), the PHTs show flat-top amplitude

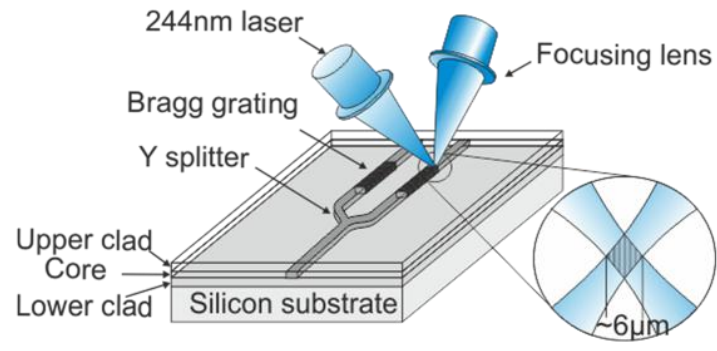

Fig. 3: Direct Grating writing demo, showing crossed beams focused on the core layer of the silica-on-silicon substrate, with the writing spot in $6 \mu \mathrm{m}$ diameter.
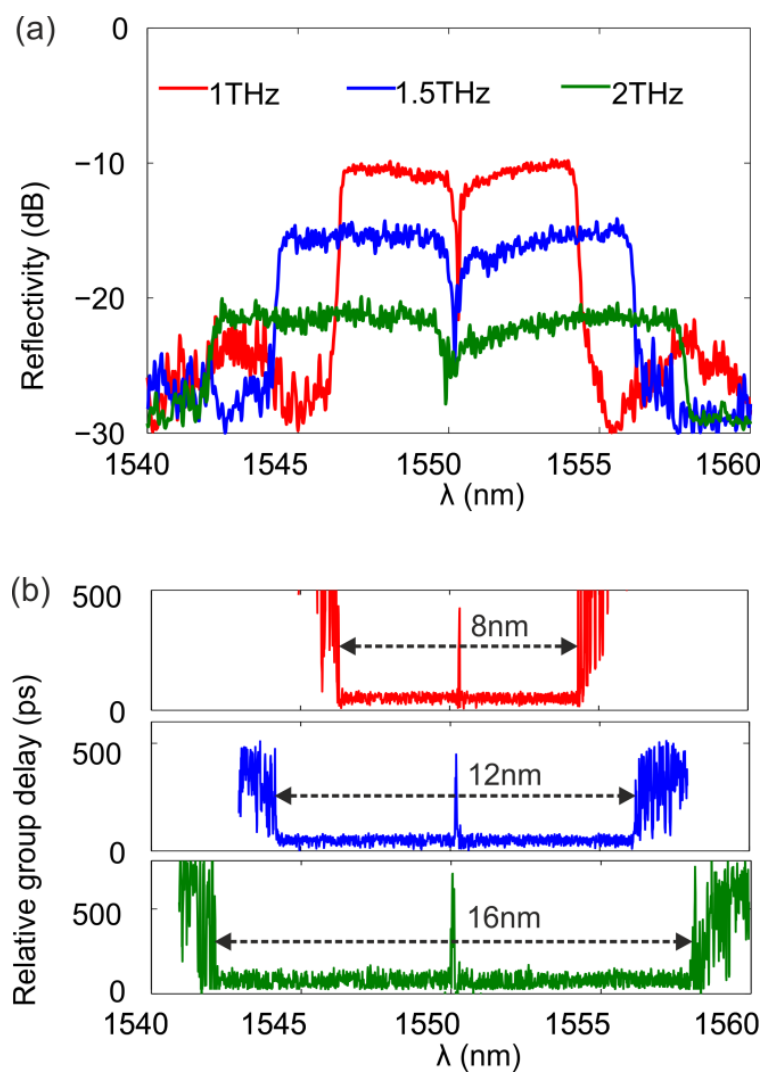

Fig. 4: Measured data: (a) the reflectivity of the proposed Bragg gratings implementing $1 \mathrm{THz}, 1.5 \mathrm{THz}$ and $2 \mathrm{THz}$ bandwidth PHTs; (b) relative group delay of the series of planar Bragg gratings. 
responses, with $<1 \mathrm{~dB}$ ripples for $1 \mathrm{THz}$ bandwidth. Figure $4(\mathrm{~b})$ indicates the $\sim \pi$ phase shift at the central dip of the amplitude spectra. We can use the active phase control, e.g. thermal tuning ${ }^{3}$, to compensate any PHT phase error. The series of initial proof-of-concept devices demonstrate $8 \mathrm{~nm}(1 \mathrm{THz}), 12 \mathrm{~nm}(1.5$ $\mathrm{THz})$ and $16 \mathrm{~nm}(2 \mathrm{THz})$ operation bandwidth PHTs.

The silica-on-silicon planar format was employed for an all-optical SSB filter ${ }^{3}$, composed of an X-coupler, a flat-top reflector and the PHT. A sinc-apodized Bragg grating was utilized as the flat-top reflector with the same length and similar bandwidth to the PHT. The reflected optical signal from the two gratings undergoes constructive and destructive interference, thereby suppressing one side band and enhancing the other. The relative optical phase difference of the two signals can be finetuned by a small micro heater present on one arm of the device ${ }^{3}$. The X-coupler length was $\sim 7.5 \mathrm{~mm}$, with crossing angle 1.8 degrees, yielding a balanced coupling ratio. The interferometric structure is schematically shown in Fig. 5(a). (a)

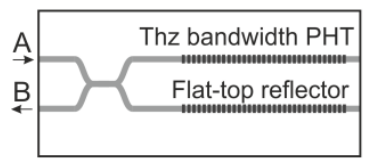

(c)

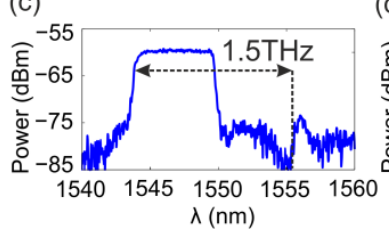

(d)

(b)

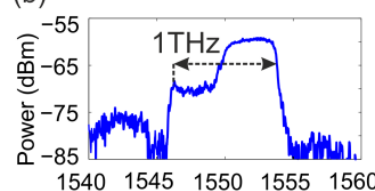

(d) $\quad \lambda(\mathrm{nm})$

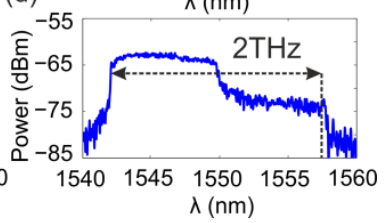

Fig. 5:(a) The principle scheme of the integrated all-optical SSB filter device; (b)-(d) Output optical power spectra at port $\mathrm{B}$, with $1 \mathrm{THz}, 1.5 \mathrm{THz}$ and $2 \mathrm{THz}$ band-width PHTs respectively, when optical broadband signal is coupled into port $A$ and received from port $B$.

Figures 5(b)-(d) show the output optical power spectra directly from port $\mathrm{B}$ of the device, incorporating the $1 \mathrm{THz}, 1.5 \mathrm{THz}$ and $2 \mathrm{THz}$ bandwidth PHTs respectively, with input at port $A$, measured using an OSA and a broadband ASE source. For example in Fig. 5(c) of the 1.5 $\mathrm{THz} \mathrm{PHT}$ scenario, optical signals among the spectral band below the central wavelength (1544-1550nm) have a ripple-less pass band while signals within the other band (1550$1556 \mathrm{~nm}$ ) are eliminated, and the power differs $\sim 20 \mathrm{~dB}$. The microwave signal operation for SSB filtering is not performed, due to the limitation of the available microwave characterization setup and not the device. Figure 5(d) indicates the operation bandwidth of the all-optical SSB filter approaches $2 \mathrm{THz}$. The total insertion loss of the actual devices were $\sim 25 \mathrm{~dB}$ due to coupling loss, propagation loss in the X-coupler and low grating reflectivity. This can be decreased by reducing the directional coupler propagation loss and using stronger gratings.

\section{Conclusions}

In summary, for the first time to our knowledge we have experimentally demonstrated a series of $\mathrm{THz}$ bandwidth photonic Hilbert transformers based on planar Bragg gratings. PHT devices displaying up to $2 \mathrm{THz}$ operation bandwidths have been fabricated via the direct UV grating writing technique. This fabrication technique allows precise engineering of the amplitude and phase response of the gratings with complex structures at micron level. The PHTs were also integrated with couplers and flat-top reflectors to perform ultra wideband all-optical SSB filters with up to $2 \mathrm{THz}$ bandwidth. These devices would allow processing of ultra-wide optical signals with terahertz bandwidths.

\section{Acknowledgements}

This work was supported by Engineering and Physical Sciences Research Council (EPSRC), UK and China Scholarship Council, China.

\section{References}

[1]. S. L. Hahn, the Transforms and Applications Handbook, 2nd ed., CRC Press, (2000).

[2]. H. Emami et al., Opt. Express, 16, 13707 (2008).

[3]. C. Sima et al., Opt. Lett., 38, 727 (2013).

[4]. C. Sima et al., Front. Optoelectron., 6, 78 (2013).

[5]. X. Wang et al., Proc. APCC, 622 (2009).

[6]. M. Asghari et al., Opt. Lett., 34, 334 (2009).

[7]. M. Li et al., Photon. Technol. Lett., 22,1559 (2010).

[8]. L. Zhuang et al., Opt. Express, 20, 26499 (2012).

[9]. R. Ashrafi et al., Opt. Lett., 37, 2604 (2012).

[10]. G. D. Emmerson et al., Electron. Lett., 38, 1531 (2002).

[11]. R. Hui et al., Fiber optic measurement techniques, Academic Press (2009). 